\title{
Prevalence of Depression in Patients with MDR TB
}

\author{
Prakash Jha ${ }^{1}$, Rahul Mishra ${ }^{2}$, Rakesh Ghildiyal ${ }^{3}$ \\ ${ }^{1}$ Resident \\ ${ }^{2}$ Senior Resident \\ ${ }^{3}$ Professor \& Head of Department \\ Department of Psychiatry, Mahatma Gandhi Mission Medical College, Kamothe, Navi-Mumbai
}

Corresponding author: Prakash Jha

Email-ishuprakash25@gmail.com

\begin{abstract}
Background and Objectives: Despite being preventable, treatable, and curable, tuberculosis (TB) remains a leading cause of mortality worldwide, with $95 \%$ of deaths occurring in low-income countries. There is limited information on the joint effect of TB and depression on health status of general population. The aim of this study is to assess the prevalence of Depression in patients of MDR TB and to study the demographic constitution and related factors of the patients with MDR TB.

Methods: This study was cross sectional survey conducted to study the prevalence of Depression in patients of MDR TB referred for psychiatric evaluation. Data was collected over a period of 3 month(July 18 to September 18) and study setting was the outpatient Department of Psychiatry at Mahatma Gandhi Missions Medical College, Kamothe, Navi Mumbai.

Results: It was found that depression was found in $52 \%$ of the patients ranging from mild to moderate and severe depression using PHQ 9 questionnaire

Conclusion: Depressive and anxiety symptoms are common among PTB patients, especially those with MDR TB. Screening for depression and other psychiatric illness is essential for identifying patients who require further psychosocial assessment and support.
\end{abstract}

Keywords: Depression, MDR TB, screening, psychiatric evaluation.

(Paper received $-10^{\text {th }}$ November 2018, Peer review completed $-10^{\text {th }}$ December 2018)

(Accepted $-14^{\text {th }}$ December 2018)

\section{INTRODUCTION}

Despite being preventable, treatable, and curable, tuberculosis (TB) remains a leading cause of mortality worldwide, with $95 \%$ of deaths occurring in low-income countries [1]. There is limited information on the joint effect of TB and depression on health status of general population [2]. In individuals appropriately treated for multidrug-resistant tuberculosis, the success rate is only about $50 \%$ worldwide because of factors such as low drug effectiveness, lengthy and toxic regimens that are difficult to complete, and high rates of prevalent and acquired resistance to second-line drugs [3]. The psychosocial issues often complicate MDR$\mathrm{TB}$, because of the complex and long duration of treatment [4]. Depression is a common comorbid condition for patients with tuberculosis (TB), and is associated with higher morbidity and mortality, antibiotic drug resistance, and community transmission. Depressed individuals with TB are less likely to seek care promptly, if at all, and once in treatment are significantly less likely to take medications consistently and/or completely. These treatment irregularities can lead to drug resistance, morbidity and mortality. Therefore, depression may be an unrecognized driver of the TB and multidrug resistant TB (MDR-TB) epidemics. MDR-TB treatment is significantly more expensive, takes approximately four times as long to complete, and produces 
acute physical and psychiatric side effects, which makes treatment adherence and completion a considerable challenge [5]. India has the second highest total number of estimated MDR TB cases $(99,000)$ in 2008, after China (100,000 cases) (WHO, 2010b) [6]. The aim of this study was to assess the prevalence and nature of depression in patients with MDR-TB

\section{METHODOLOGY}

It was a cross sectional survey conducted to study the prevalence of Depression in patients of MDR TB referred for psychiatric evaluation. Data was collected over a period of 3 months (July 18 to September 18) and study setting was the outpatient Department of Psychiatry at Mahatma Gandhi Missions Medical College, Kamothe, Navi Mumbai. All MDR TB patients referred from department of Respiratory Medicine for Psychiatric evaluation to the Out Patient Department of Psychiatry at MGM Hospital, Kamothe, Navi Mumbai were eligible to participate in the study. Patients having severe psychiatric or medical illness, mental retardation or sensory impairments rendering them uncooperative for assessments and consent were excluded from the study. Informed consent of the patients were taken. Patients were administered a semi structured proforma to collect the demographic data, also these patients were administered, Public Health Questionnaire 9 to assess depression.

Public Health Questionnaire 9: The PHQ-9 is a component of the longer Patient Health Questionnaire which offers psychologists concise, self-administered tools for assessing depression. They incorporate DSMIV depression criteria with other leading major depressive symptoms into a brief self-report instruments that are commonly used for screening and diagnosis, as well as selecting and monitoring treatment. PHQ-9 scores $>10$ had a sensitivity of $88 \%$ and a specificity of $88 \%$ for Major Depressive Disorder. Internal consistency of the PHQ-9 has been shown to be high. A study involving two different patient populations produced Cronbach alphas of 0.86 and 0.89 [7]. Data was entered in Excel sheet and Data collected was analyzed for gender profile and demographic factors using SPSS version 23. Institutional Ethics clearance was obtained.

\section{RESULTS}

A total of 50 patients were assessed for psychiatric morbidities referred for psychiatric assessment from Department of Chest and TB, all of these patients were patients with a confirmed diagnosis of MDR TB and were going to be started or had been started on Capsule Cycloserine.

Out of 50 patients $29(58 \%)$ were males and $21(42 \%)$ were females. $38(76 \%)$ out of 50 patients were married and $10(20 \%)$ were unmarried, one was a widow and 1 was a divorcee. Educational profile of the patients consisted of $40 \%$ were graduates, $18 \%$ had received secondary education, $18 \%$ were educated upto primary school, $14 \%$ of the patients were post graduates and $10 \%$ were illiterates. Family makeup of the patients consisted of $78 \%$ lived in Nuclear family, $14 \%$ lived in a joint family and $8 \%$ lived in extended nuclear family. Occupational profile of the patients consisted of $38 \%$ were skilled workers, $36 \%$ were unskilled workers, $16 \%$ were students and $10 \%$ were unemployed. According to Modified BG prasad classification for socioeconomic status, $38 \%$ of Patients were belonging to upper class, $32 \%$ were of upper middle class and $30 \%$ belonging to Lower middle class.

\section{PHQ 9 Profile}

In our study we found that depression was found in $26(52 \%)$ of the patients ranging from mild to moderate and severe depression using PHQ 9 questionnaire. Out of this 52\%, 24\% patients had mild depression, $24 \%$ had moderate depression and $4 \%$ had moderately severe depression.

\section{DISCUSSION}

MDR TB is posing a serious threat to the health system of India with an ever-increasing number of newly diagnosed cases of TB and also an outbreak of sorts of these cases being diagnosed as a fresh case of MDR TB as well. Majority of these patients have to undergo a long treatment regimen and have to take up to 10 to 15 medications both through oral route and injectable forms. Current study was initiated as there was an MOU signed by the Government of Maharashtra to look into the psychiatric morbidities and also to provide 
counselling for these patients to start the medications and also to improve treatment adherence and decrease drop out cases.

Researchers in their study of Burden and presentation of depression among newly diagnosed individuals with TB in primary care settings in Ethiopia found that the overall prevalence of probable depression was $54.0 \%$ which is higher than reported in a study that used the depression component of the hospital anxiety and depression scale in which the prevalence was $43.4 \%$ [8].

Kumar and others found psychiatric morbidities amongst $74 \%$ of the patients employing GHQ12 and also these patients benefited from psychiatric intervention and psychoeducation [9]. In our study we found that depression was found in $52 \%$ of the patients ranging from mild to moderate and severe depression using PHQ 9 questionnaire.

In a study relating to depression in TB patients, researchers found the prevalence of TB more in males than females. Also, they found that prevalence was higher amongst low socioeconomic strata [10]. In our study we found that TB was present in $58 \%$ of males compared to $42 \%$ in females also in our study majority of patients belonged to upper class $38 \%$, upper middle class $32 \%$ and lower middle class $30 \%$ this difference may be due to urban setting of our centre and higher per capita income in the Indian cities.

In the study of 86 patients with TB done by Ana Paula Ceré dos Santos et al, the mean age was 44.6 years, $27(31.4 \%)$ patients met the study criteria for depression and $69.8 \%$ of them were male [11]. In our study average age of patients was 36 years, $52 \%$ met the study criteria for depression with prevalence of depression more in males than in females.

\section{Limitation of the Study}

In our study we only studied one parameter i.e., Depression, more parameters could be included in the future study to have a complete profile of psychiatric morbidity amongst patients with TB.

Follow up studies could help in assessing effects of psychiatric medication in relation to drop out rates and treatment compliance.

\section{Strength of Study}

PHQ 9 has been widely used to study the prevalence of depression in the clinical setting as it is easy to use and a short questionnaire has good inter-test reliability and validity. Psychiatric co morbidities are high amongst TB patients.

\section{CONCLUSION}

Depressive and anxiety symptoms are common among PTB patients, especially those with MDR TB. Screening for depression and other psychiatric illness is essential for identifying patients who require further psychosocial assessment and support. Psychiatric assessment can help in increasing the treatment compliance of the patient and also in getting these patients over the difficult phase of treatment initially in which patients may experience sadness, hopelessness, helplessness and worthlessness. Treatment protocol for MDR TB includes capsule Cycloserine which is known to cause depression and psychosis which may lead to higher drop out amongst patients and also may add to significant mortality. Family member need to be counselled and psychoeducated as the usual treatment duration is long and testing at times. Good family support and awareness amongst the care givers also significantly reduces the risk of disease transmission and better prognosis for the patients.

\section{REFERENCES}

1. Mason PH, Sweetland AC, Fox GJ, Halovic S, Nguyen TA, Marks GB. Tuberculosis and mental health in the Asia-Pacific. Australas Psychiatry 2016;24(6):553-5.

2. Koyanagi A, Vancampfort D, Carvalho AF, DeVylder JE, Haro JM, Pizzol D, et al. Depression comorbid with tuberculosis and its impact on health status: cross-sectional analysis of community-based data from 48 low- and middle-income countries. BMC Med 2017;15(1):209.

3. Kendall EA, Fojo AT, Dowdy DW. Expected effects of adopting a 9 month regimen for multidrug-resistant tuberculosis: a population modelling analysis. Lancet Respir Med 2017;5(3):191-9. 
4. Thomas BE, Shanmugam P, Malaisamy M, Ovung S, Suresh C, Subbaraman R, et al. Psycho-Socio-Economic Issues Challenging Multidrug Resistant Tuberculosis Patients: A Systematic Review. PLoS One. 2016;11(1):e0147397.

5. Sweetland A, Oquendo M, Wickramaratne P, Weissman M, Wainberg M. Depression: a silent driver of the global tuberculosis epidemic. World Psychiatry 2014;13(3):325-6.

6. Institute of Medicine. Facing the Reality of Drug-Resistant Tuberculosis in India: Challenges and Potential Solutions: Summary of a Joint Workshop by the Institute of Medicine, the Indian National Science Academy, and the Indian Council of Medical Research. Washington (DC): The National Academies Press; 2012. https://doi.org/10.17226/13243.

7. Zhang YL, Liang W, Chen ZM, Zhang HM, Zhang JH, Weng XQ, et al. Validity and reliability of Patient Health Questionnaire-9 and Patient Health Questionnaire-2 to screen for depression among college students in China. Asia Pacific Psychiatr 2013;5(4):268-75.

8. Ambaw F, Mayston R, Hanlon C, Alem A. Burden and presentation of depression among newly diagnosed individuals with TB in primary care settings in Ethiopia. BMC Psychiatry 2017;17(1):57.

9. Kumar K, Kumar A, Chandra P, Kansal HM. A study of prevalence of depression and anxiety in patients suffering from tuberculosis. J Family Med Prim Care 2016;5(1):150-3.

10. Internet Applications.emro.who.int. $2018 \quad$ [cited $30 \quad$ October 2018 ]. Availablefrom:http://applications.emro.who.int/imemrf/Ann_Punjab_Med_Coll/Ann_Punjab_Med_Coll_ 2010_4_2_133_137.pdf

11. Dos Santos AP, Lazzari TK, Silva DR. Health-Related Quality of Life, Depression and Anxiety in Hospitalized Patients with Tuberculosis. Tuberc Respir Dis (Seoul) 2017;80(1):69-76.

$* * * * * * * * * * * * * * * * * * * * * * * * * * * * * * * * * * * *$

Acknowledgements - Nil

Conflict of Interest - Nil

Funding - Nil 